\title{
ON SYMMETRIC SQUARE VALUES OF QUADRATIC POLYNOMIALS
}

\author{
ENRIQUE GONZÁLEZ-JIMÉNEZ AND XAVIER XARLES
}

\begin{abstract}
We prove that there does not exist a non-square quadratic polynomial with integer coefficients and an axis of symmetry which takes square values for $N$ consecutive integers for $N=7$ or $N \geq 9$. At the opposite, if $N \leq 6$ or $N=8$ there are infinitely many.
\end{abstract}

\section{INTRODUCTION}

In this note we are dealing with the following problem. Given a degree two polynomial $f(x)=a x^{2}+b x+c \in \mathbb{Z}[x]$ which is not a square of a degree one polynomial, how many consecutive integers values $f(i)$ can be squares in $\mathbb{Z}$ ? This problem has been considered by D. Allison in [1 and 2], who found infinitely many examples with eight consecutive values, and by A. Bremner in [3], who found more examples with seven consecutive values.

The examples found by Allison are all by polynomials which are symmetric with an axis of symmetry midway between two integers. This means that, after some easy translation, all the examples are of the form $f(x)=$ $a\left(x^{2}+x\right)+c$ and the values are $f(i)$ for $i=-3,-2,-1,0,1,2,3$ and 4 . This result was obtained by translating the problem to computing rational points on some elliptic curve which has rank one.

On the other hand, Bremner in [3] shows that there does not exists any example which is symmetric with an axis of symmetry about an integral value and with 7 values, by showing that these examples would be described by rational points in some rank zero elliptic curve, which has 12 points, all corresponding to the polynomial $f(x)$ being the square of a polynomial.

In the same paper, Bremner asks if there are examples as the ones found by Allison, but with 10 consecutive squares. The problem translates to finding all the rational points of a genus 5 curve, a fact already noticed by Allison and by Bremner. He conjectures that there should be no such example.

Date: April 2, 2022.

2000 Mathematics Subject Classification. Primary: 11G30, 11D45; Secondary: 14H25.

Key words and phrases. squares, quadratic polynomials, covering collections, elliptic Chabauty.

The first author was supported in part by grants MTM 2009-07291 (Ministerio de Educación y Ciencia, Spain) and CCG08-UAM/ESP-3906 (Universidad Autonóma de Madrid-Comunidad de Madrid, Spain). The second author was partially supported by the grant MTM2009-10359. 
In this note we prove this conjecture, and so, combining with the results of Bremner and Allison, we get the following theorem.

Theorem 1. Let $N$ be a positive integer and $\mathcal{B}_{N}$ the set consisting of nonsquare quadratic polynomials $f(x)=a x^{2}+b x+c \in \mathbb{Z}[x]$ that takes square values for $N$ consecutive integers values of $x, r, r+1, \ldots, r+N-1$ and $f(r)=f(r+N-1)$, for some $r \in \mathbb{Z}$. Then

$$
\# \mathcal{B}_{N}=\left\{\begin{array}{cl}
\infty & \text { if } N \leq 6 \text { or } N=8 \\
0 & \text { if } N=7 \text { or } N \geq 9 .
\end{array}\right.
$$

To show this result we will use similar techniques as the one we use in 11 to study the arithmetic progressions of squares over quadratic fields. In fact, the problem we study here is in some sense a generalization to higher dimension of the old result by Fermat about arithmetic progressions of squares, and the problems in [11] and in [14] are generalizations to higher degree (in the sense of the field of numbers involved). However, we can say almost nothing about if there exists a maximum number of consecutive square values taken by a non-square quadratic polinomial, or even if this number is 8 , as the known examples suggest.

\section{Translation to Geometry}

Fix a polynomial $f(x)=a x^{2}+b x+c \in \mathbb{Z}[x]$ which takes square values for $N$ consecutive integers values of $x$, of the form $r, r+1, \ldots, r+N-1$, and $f(r)=f(r+N-1)$. Suppose that $N$ is even. Then, after translation by $-r-N / 2$, we can suppose that $r=-N / 2$. We get then that $f(x)$ has the form $f(x)=a\left(x^{2}+x\right)+c$, and we are asking to have $f(i)=x_{i}^{2}$ for $i=0, \ldots, N / 2-1$ and $x_{i} \in \mathbb{Z}$.

Now, suppose that $N=10$. The conditions we get from $f(i)=x_{i}^{2}$ for $i=0, \ldots, 4$ are given by the following equations:

$$
C:\left\{\begin{array}{l}
2 x_{0}^{2}-3 x_{1}^{2}+x_{2}^{2}=0 \\
5 x_{0}^{2}-6 x_{1}^{2}+x_{3}^{2}=0 \\
9 x_{0}^{2}-10 x_{1}^{2}+x_{4}^{2}=0
\end{array}\right.
$$

which determine a genus 5 curve $C$ in $\mathbb{P}^{4}$. Any point $P:=\left[x_{0}: x_{1}: x_{2}: x_{3}:\right.$ $x_{4}$ ] of this curve defined over $\mathbb{Q}$ will give us a polynomial $f(x)$ as before, by setting $c=x_{0}^{2}$ and $a=\left(x_{1}^{2}-x_{0}^{2}\right) / 2$. Observe that the pairs $(a, c)$ are well defined modulo multiplication by a square number, which will produce the same polynomial but multiplied by a square number, a case that we consider equivalent.

Now, the solutions given by $P=[ \pm 1: \pm 1: \pm 1: \pm 1: \pm 1]$ correspond to the case $a=0$, so the polynomial is in fact constant. There are also the solutions given by $P=[ \pm 1: \pm 3: \pm 5: \pm 7: \pm 9]$, which correspond to the case $a=4$ and $c=1$, so $f(x)=(2 x+1)^{2}$. Our aim will be to show that these are the only rational points. 
First of all, observe that the curve $C$ has degree 2 maps $\Phi_{n}$ to five distinct elliptic curves $E_{n}$, for $n=0,1,2,3,4$. They can be described easily as intersection of two quadrics in $\mathbb{P}^{3}$, by taking first two of the three quadrics describing $C$, which give three of them, and transforming the equations in order to get more quadratic forms involving only three variables, which gives the other two. We denote by $F_{n}$ to the genus one curve obtained by removing the variable $x_{n}$ for $n=0,1,2,3,4$. We have obtained the following equations for such a curves:

$$
\begin{array}{lll}
F_{0}: x_{1}^{2}=16 t^{4}-144 t^{3}+340 t^{2}-252 t+49, & t=\frac{x_{4}+x_{2}}{x_{4}-x_{3}}, \\
F_{1}: x_{0}^{2}=16 t^{4}-160 t^{3}+384 t^{2}-280 t+49, & t=\frac{x_{4}+x_{2}}{x_{4}-x_{3}}, \\
F_{2}: x_{4}^{2}=36 t^{4}+96 t^{3}-236 t^{2}+80 t+25, & t=\frac{x_{3}+x_{1}}{x_{3}-x_{0}}, \\
F_{3}: x_{1}^{2}=100 t^{4}-360 t^{3}+472 t^{2}-252 t+49, & t=\frac{x_{4}+x_{2}}{x_{4}-x_{0}}, \\
F_{4}: x_{2}^{2}=36 t^{4}-72 t^{3}+72 t^{2}-60 t+25, & t=\frac{x_{3}+x_{1}}{x_{3}-x_{0}} .
\end{array}
$$

The second column is in fact equivalent to the forgetful map $\varrho_{n}: C \longrightarrow F_{n}$. Observe that there is always a quadratic form involving only three of the variables $x_{n}$, for any choice of them. The curve $F_{n}$ is isomorphic to the one given by equations not involving the variable $x_{n}$.

All these genus one curves have rational points over $\mathbb{Q}$, therefore they are elliptic curves over $\mathbb{Q}$. A Weierstrass model of the quartic $F_{n}$ is denoted by $E_{n}, n=0,1,2,3,4$ :

$$
\begin{aligned}
& E_{0}: y^{2}=x(x-8)(x+27), \\
& E_{1}: y^{2}=x(x-12)(x+30), \\
& E_{2}: y^{2}=x(x+4)(x+54), \\
& E_{3}: y^{2}=x(x-7)(x+20), \\
& E_{4}: y^{2}=x(x-12)(x-15) .
\end{aligned}
$$

Using the labeling of the Cremona's tables [9], one can check that $E_{0}=$ $1680 \mathrm{G} 2, E_{1}=20160 \mathrm{BG} 2, E_{2}=960 \mathrm{H} 2, E_{3}=840 \mathrm{H} 2$ and $E_{4}=360 \mathrm{E} 2$.

So, if one of such elliptic curves have a finite number of rational points, then the problem of computing $C(\mathbb{Q})$ becomes easy. Now, it is a straightforward computation to check that the torsion subgroup of $E_{n}(\mathbb{Q})$ is isomorphic to $\mathbb{Z} / 2 \mathbb{Z} \oplus \mathbb{Z} / 2 \mathbb{Z}$. On the other hand, the set $\Phi_{n}([ \pm 1: \pm 1: \pm 1: \pm 1: \pm 1])$ has cardinality eight. Therefore the rank of $E_{n}(\mathbb{Q})$ is greater than one, that is, we cannot use these argument to determine $C(\mathbb{Q})$. In fact, we can easily compute by descent (or, better, using some algebraic computational system like Magma or Sage, or even better, checking at the Cremona's tables), that $\operatorname{rank}_{\mathbb{Z}} E_{n}(\mathbb{Q})=1$ for $n \neq 1$ and $\operatorname{rank}_{\mathbb{Z}} E_{1}(\mathbb{Q})=2$.

Another approach is the Chabauty's method. This could be used when the Jacobian of the curve has rank less than the genus of the curve. But in this case the rank of $\operatorname{Jac}(C)$ is greater than 5 , the genus of $C$, since $\operatorname{Jac}(C)$ is $\mathbb{Q}$-isogenous to the product of $E_{n}$, for $n=0,1,2,3,4$. So we cannot apply 
this method. Other methods, like the Manin-Drinfeld's method, cannot be applied either.

\section{Two Descent and Covering Collections}

In order to actually compute the rational points in the curve $C$, we will apply the covering collections technique, as developed by Coombes and Grant [8], Wetherell [15] and others, and specifically a modification of what is now called the elliptic Chabauty method developed by Flynn and Wetherell in [10] and by Bruin in [4].

The method has two parts. Suppose we have a curve $C$ over a number field $K$ and an unramified map $\chi: C^{\prime} \rightarrow C$ of degree greater than one and may be defined over a finite extension $L$ of $K$. We consider the distinct unramified coverings $\chi^{(s)}: C^{\prime(s)} \rightarrow C$ formed by twists of the given one, and we get that

$$
C(K)=\bigcup_{s} \chi^{(s)}\left(\left\{P \in C^{(s)}(L): \chi^{(s)}(P) \in C(K)\right\}\right),
$$

the union being disjoint. Only a finite number of twists do have rational points, and the finite (larger) set of twists having points locally everywhere can be explicitly described. The first part is to compute this set of twists, and the second to compute the points $P \in C^{\prime(s)}(L)$ such that $\chi^{(s)}(P) \in C(K)$. This second part depends on having a nice quotient of the curves $C^{\prime(s)}$, for example a genus one quotient, where it is possible to do the computations. In this section we will concentrate on the first part.

The coverings we are going to consider are Galois coverings with Galois group isomorphic to $(\mathbb{Z} / 2 \mathbb{Z})^{2}$. This coverings are in principle easy to construct. One only needs to have an isogeny map from an abelian variety $A$ to the jacobian $\operatorname{Jac}(C)$ of the curve $C$ with kernel isomorphic, as group scheme, to the group $(\mathbb{Z} / 2 \mathbb{Z})^{2}$. Since in our case the jacobian $\operatorname{Jac}(C)$ is isogenous to a product of elliptic curves $E_{i}$, they can be constructed by choosing two such elliptic curves and one degree two isogeny in each of them.

Moreover, the elliptic curves $E_{i}$ have all they 2-torsion points defined over $\mathbb{Q}$, hence the coverings we are searching for will be defined over $\mathbb{Q}$. On the other hand, the genus one quotients of such coverings that we will use in the next section are, in general, not defined over $\mathbb{Q}$, but in a quadratic or in a biquadratic extension. The way we will construct the coverings, by using a factorization of the quartic polynomials, will give us directly also the genus one quotient and the field where it is defined.

In order now to construct the coverings of the curve $C$, we first rewrite the equations of the curve in the following form:

$$
C:\left\{\begin{array}{l}
y^{2}=q(t)=36 t^{4}-72 t^{3}+72 t^{2}-60 t+25 \\
z^{2}=p(t)=36 t^{4}+96 t^{3}-236 t^{2}+80 t+25
\end{array}\right.
$$

This model has two natural maps to the genus one curves $F_{4}: y^{2}=q(t)$ and $F_{2}: z^{2}=p(t)$, whose jacobians are $E_{4}$ and $E_{2}$ respectively. 
First, we concentrate on the unramified degree two coverings of the genus one curve given by a quartic model. If $F$ is such a genus one curve defined over a field $K$, given by the equation $y^{2}=r_{1}(x) r_{2}(x)$, where $r_{1}(x)$ and $r_{2}(x)$ are degree two polynomials defined over an extension $L$ of $K$, we consider the degree two unramified covering $\chi: F^{\prime} \rightarrow F$ with affine part in $\mathbb{A}^{3}$ given by the zeros of the polynomials $y_{1}^{2}=r_{1}(x)$ and $y_{2}^{2}=r_{2}(x)$, the map given by $\chi\left(x, y_{1}, y_{2}\right)=\left(x, y_{1} y_{2}\right)$. For any $\delta \in L^{*}$, we consider the curve $F^{\prime(\delta)}$ given by the equations $\delta y_{1}^{2}=p_{1}(x)$ and $\delta y_{2}^{2}=p_{2}(x)$, and the map to $F$ defined by $\chi^{(\delta)}\left(x, y_{1}, y_{2}\right)=\left(x, y_{1} y_{2} / \delta^{2}\right)$. Then $F^{\prime(\delta)}$ are all the quadratic twists of $F^{\prime}$, and there exists a finite set $\Delta_{L}(\chi) \subset L^{*}$ such that

$$
F(K) \subseteq \bigcup_{\delta \in \Delta_{L}(\chi)} \chi^{(\delta)}\left(\left\{\left(x, y_{1}, y_{2}\right) \in F^{\prime(\delta)}(L): x \in K \text { or } x=\infty\right\}\right) .
$$

First, we consider the case of $F_{2}$, given as $y^{2}=p(t)$, where

$$
p(t)=36 t^{4}+96 t^{3}-236 t^{2}+80 t+25=\left(6 t^{2}-4 t-1\right)\left(6 t^{2}+20 t-25\right) .
$$

Lemma 2. Consider the degree two covering defined over $\mathbb{Q}$ given by

$$
F_{2}^{\prime}:\left\{\begin{array}{l}
z_{1}^{2}=p_{1}(t)=6 t^{2}-4 t-1, \\
z_{2}^{2}=p_{2}(t)=6 t^{2}+20 t-25,
\end{array}\right.
$$

together with the natural map $\psi_{2}: F_{2}^{\prime} \rightarrow F_{2}$ given by $\psi_{2}\left(t, z_{1}, z_{2}\right)=\left(t, z_{1} z_{2}\right)$. Then $\Delta_{\mathbb{Q}}\left(\psi_{2}\right):=\{ \pm 1, \pm 6\}$, hence

$$
F_{2}(\mathbb{Q}) \subseteq \bigcup_{\delta \in\{ \pm 1, \pm 6\}} \psi_{2}^{(\delta)}\left(\left\{\left(t, z_{1}, z_{2}\right) \in F_{2}^{\prime(\delta)}(\mathbb{Q})\right\}\right) .
$$

Proof. It is easy to show and well-known that $\psi_{2}$ is an unramified degree two covering of $F_{2}$. Since $F_{2}^{\prime}(\mathbb{Q}) \neq \emptyset$, because it contains the point $P^{\prime}:=$ $(1,1,1)$, we can identify $F_{2}^{\prime}$ with an elliptic curve $E_{2}^{\prime}$, by sending this point $P^{\prime}$ to $O$, and, identify $F_{2}$ with the elliptic curve $E_{2}$ by sending the point $P:=\chi\left(P^{\prime}\right)=(1,1)$ to the point $O$. We get then an unramified degree two covering $\phi_{2}: E_{2}^{\prime} \rightarrow E_{2}$, which must be a degree 2 isogeny. With appropriate choices of the identifications, we can get this isogeny in the standard form (see, for example, [12, III.4.5] or [7, §8.2]). After some computations we get the map $\phi_{2}: E_{2}^{\prime} \rightarrow E_{2}$ defined by

$$
\phi_{2}(x, y)=\left(\frac{y^{2}}{4 x^{2}}, \frac{y\left(x^{2}-2500\right)}{8 x^{2}}\right),
$$

where $E_{2}: y^{2}=x(x-4)(x+54)$ and $E_{2}^{\prime}: y^{2}=x\left(x^{2}-116 x+2500\right)$.

Now, the quadratic twists $F_{2}^{\prime(\delta)}$ which locally have rational points correspond to the elements of the Selmer group $\operatorname{Sel}\left(\phi_{2}\right)$. After identifying $\operatorname{Sel}\left(\phi_{2}\right)$ with a subgroup of $\mathbb{Q}^{*} /\left(\mathbb{Q}^{*}\right)^{2}$ in the standard way, the identification sends $\delta \in \mathbb{Q}^{*}$ to its class modulo squares. A standard 2-descent calculation gives that $\operatorname{Sel}\left(\phi_{2}\right)=\{ \pm 1, \pm 6\}$. But now, by using that $E_{2}(\mathbb{Q})$ contains the points $(0,0),(-54,0)$ and $(36,-360)$, one can see that all the elements of 
the Selmer group $\operatorname{Sel}\left(\phi_{2}\right)$ correspond to elements of $E(\mathbb{Q})$. These are exactly the $\delta$ 's such that $F_{2}^{\prime(\delta)}(\mathbb{Q}) \neq \emptyset$.

Now we consider the curve $F_{4}$, given as $y^{2}=q(t)$, where $q(t)=36 t^{4}-$ $72 t^{3}+72 t^{2}-60 t+25$. Observe that the polynomial $q(t)$ is irreducible over $\mathbb{Q}$, but it factorizes over some quadratic extensions as product of two degree 2 polynomials. Over $\mathbb{Q}(\sqrt{6})$ we have

$q(t)=(5+2 \sqrt{6})\left(6 t^{2}+2 \sqrt{6} t+5-6 \sqrt{6}\right) \cdot(5-2 \sqrt{6})\left(6 t^{2}-2 \sqrt{6} t+5+6 \sqrt{6}\right)$.

Lemma 3. Consider the degree two covering defined over $\mathbb{Q}$ given by

$$
F_{4}^{\prime}:\left\{\begin{array}{l}
y_{1}^{2}=q_{1}(t)=(5+2 \sqrt{6})\left(6 t^{2}+2 \sqrt{6} t+5-6 \sqrt{6}\right), \\
y_{2}^{2}=q_{2}(t)=(5-2 \sqrt{6})\left(6 t^{2}-2 \sqrt{6} t+5+6 \sqrt{6}\right),
\end{array}\right.
$$

together with the natural map $\psi_{4}: F_{4}^{\prime} \rightarrow F_{4}$ given by $\psi_{4}\left(t, y_{1}, y_{2}\right)=\left(t, y_{1} y_{2}\right)$. Then $\Delta_{\mathbb{Q}(\sqrt{6})}\left(\psi_{4}\right):=\{1,2,5,10\}$, hence

$$
F_{4}(\mathbb{Q}) \subseteq \bigcup_{\delta \in\{1,2,5,10\}} \psi_{4}^{(\delta)}\left(\left\{\left(t, y_{1}, y_{2}\right) \in F_{4}^{\prime(\delta)}(L): t \in \mathbb{Q} \text { or } t=\infty\right\}\right)
$$

Proof. As in the proof of the lemma above, observe that $F_{4}^{\prime}(\mathbb{Q}(\sqrt{6}))$ contains the point $(1,1,1)$, such that $\psi_{4}(1,1,1)=(1,1) \in F_{4}(\mathbb{Q})$. Then the degree two covering $\psi_{4}: F_{4}^{\prime} \rightarrow F_{4}$ defined over $\mathbb{Q}(\sqrt{6})$ corresponds, by taking some isomorphisms to the respective jacobians, to the 2 -isogeny $\phi_{4}: E_{4}^{\prime} \rightarrow E_{4}$ defined by

$$
\phi_{4}(x, y)=\left(\frac{y^{2}}{4 x^{2}}, \frac{y\left(x^{2}-9\right)}{8 x^{2}}\right)
$$

where $E_{4}: y^{2}=x(x-12)(x-15)$ and $E_{4}^{\prime}: y^{2}=x\left(x^{2}+54 x+9\right)$, which is the dual isogeny of the 2-isogeny corresponding to the 2-torsion point $P=(0,0) \in E_{4}(\mathbb{Q})$. Now, a descent computation shows that $\operatorname{Sel}\left(\phi_{4}\right)=$ $\{1,3,2,6,5,15,10,30\}$. But observe now that two $\delta$ and $\delta^{\prime} \in \mathbb{Q}$ that are equivalent modulo squares over $\mathbb{Q}(\sqrt{6})^{*}$ give isomorphic coverings $\psi_{4}^{(\delta)}$. Hence we need only to consider the set $\Delta_{\mathbb{Q}(\sqrt{6})}\left(\psi_{4}\right)$ which is $\operatorname{Sel}\left(\phi_{4}\right)$ modulo $\left(\mathbb{Q}(\sqrt{6})^{*}\right)^{2}$, which gives the result.

We take now the unramified covering $\xi: C^{\prime} \rightarrow C$ defined by the equations:

$$
C^{\prime}:\left\{\begin{array}{l}
y_{1}^{2}=q_{1}(t)=(5+2 \sqrt{6})\left(6 t^{2}+2 \sqrt{6} t+5-6 \sqrt{6}\right), \\
y_{2}^{2}=q_{2}(t)=(5+2 \sqrt{6})\left(6 t^{2}-2 \sqrt{6} t+5+6 \sqrt{6}\right), \\
z_{1}^{2}=p_{1}(t)=6 t^{2}-4 t-1, \\
z_{1}^{2}=p_{2}(t)=6 t^{2}+20 t-25,
\end{array}\right.
$$

which is a curve of genus 17 .

The lemmata above computes the relevant twists to be consider. 
Corollary 4. The set of revelant twists is equal to

$$
\Delta:=\left\{\left(\delta_{2}, \delta_{4}\right) \in \mathbb{Q}(\sqrt{6})^{*} \mid \delta_{i} \in \Delta_{\mathbb{Q}(\sqrt{6})}\left(\phi_{i}\right), i=2,4\right\},
$$

where $\Delta_{\mathbb{Q}(\sqrt{6})}\left(\phi_{2}\right)=\{ \pm 1\}$ and $\Delta_{\mathbb{Q}(\sqrt{6})}\left(\phi_{4}\right)=\{1,2,5,10\}$, which correspond to a set of representatives in $\mathbb{Q}(\sqrt{6})$ of the image of Selmer groups of $\phi_{i}$ $(i=2,4)$ in $\mathbb{Q}(\sqrt{6})^{*} /\left(\mathbb{Q}(\sqrt{6})^{*}\right)^{2}$ via the natural maps.

Hence,

$$
C(\mathbb{Q}) \subseteq \bigcup_{\delta \in \Delta} \chi^{(\delta)}\left(\left\{\left(t, y_{1}, y_{2}, z_{1}, z_{2}\right) \in C^{\prime(\delta)}(\mathbb{Q}(\sqrt{6})): t \in \mathbb{Q} \text { or } t=\infty\right\}\right),
$$

where $C^{\prime\left(\delta_{2}, \delta_{4}\right)}$ is the curve defined by

$$
C^{\prime\left(\delta_{2}, \delta_{4}\right)}:\left\{\delta_{4} y_{1}^{2}=q_{1}(t), \delta_{4} y_{2}^{2}=q_{2}(t), \delta_{2} z_{1}^{2}=p_{1}(t), \delta_{2} z_{2}^{2}=p_{2}(t)\right\} .
$$

Proof. After the lemmata above, we only need to observe that $\Delta_{\mathbb{Q}}\left(\phi_{2}\right)=$ $\{ \pm 1, \pm 6\}$ becomes, after taking the image in $\mathbb{Q}(\sqrt{6})^{*} /\left(\mathbb{Q}(\sqrt{6})^{*}\right)^{2}$, the set $\Delta_{\mathbb{Q}(\sqrt{6})}\left(\phi_{2}\right)=\{ \pm 1\}$.

One can reduce even further the set of revelant twists to be considered by using the natural automorphisms of $C$ given by interchanging the sign of one of the coordinates (in the first model of $C$ ).

Corollary 5. Let $\tau_{i}$ be the automorphisms of $C$ given by $\tau_{i}\left(x_{i}\right)=-x_{i}$, and $\tau_{i}\left(x_{j}\right)=x_{j}$ if $j \neq i$, for $i=0,1,2,3$ and 4 , and let $\Upsilon$ be the subgroup they generate. Let $\Delta^{\prime}=\{(1,1),(-1,1)\}$. Then, for any $P \in C(\mathbb{Q})$, there exits $\tau \in \Upsilon$ and $\delta \in \Delta^{\prime}$ such that

$$
\tau(P) \in \chi^{(\delta)}\left(\left\{\left(t, y_{1}, y_{2}, z_{1}, z_{2}\right) \in C^{\prime(\delta)}(\mathbb{Q}(\sqrt{6})): t \in \mathbb{Q} \text { or } t=\infty\right\}\right),
$$

where $\chi^{( \pm 1,1)}\left(x, y_{1}, y_{2}, z_{1}, z_{2}\right)=\left(x, y_{1} y_{2}, z_{1} z_{2}\right)$.

Proof. In order to prove the result, it is enough to show that for any point $P \in C(\mathbb{Q})$, and for any $\delta_{4} \in \Delta_{\mathbb{Q}(\sqrt{6})}\left(\phi_{4}\right)$, there exists $\delta_{2} \in \Delta_{\mathbb{Q}(\sqrt{6})}\left(\phi_{2}\right)$ and $\tau \in \Upsilon$ such that $\tau(P) \in \chi^{\left(\delta_{2}, \delta_{4}\right)} C^{\prime\left(\delta_{2}, \delta_{4}\right)}(\mathbb{Q}(\sqrt{6}))$. Therefore, one can reduce to show that for any $\delta_{4} \in \Delta_{\mathbb{Q}(\sqrt{6})}\left(\phi_{4}\right)$, there exists $\tau \in \Upsilon$ such that the image of $\varrho_{2}(\tau(P))$ in $\operatorname{Sel}\left(\phi_{2}\right)$ is equal to $\delta_{2}$ modulo $\left(\mathbb{Q}(\sqrt{6})^{*}\right)^{2}$, where $\varrho_{2}: C \rightarrow F_{2}$ is the map given in section 2 .

Observe that the involutions $\tau_{i}$ for $i=0,1,3,4$ determine involutions in $F_{4}$, which in turn determine involutions $\tau_{i}^{\prime}$ in $E_{4}$ once fixed the isomorphism between $F_{4}$ and $E_{4}$. These involutions must be of the form $\tau_{i}^{\prime}(Q)=-Q+Q_{i}$ for some $Q_{i} \in E(\mathbb{Q})$, since they do have fixed points. Hence, the involutions $\tau_{i}^{\prime}$ are determined once we know the image of a single point $Q$. Thus, if we know the result for just one point $P \in C(\mathbb{Q})$, we will obtain the result for all points in $C(\mathbb{Q})$.

Take $P:=[1: 1: 1: 1: 1]$. Then one shows easily that the image of $\varrho_{2}\left(\tau_{i}(P)\right)$ in the Selmer group $\operatorname{Sel}\left(\phi_{2}\right)$ for $i=0,1,3$, together with $\varrho_{2}(P)$, covers all the group, which proves the result. 
Remark 6 . An easy computation by using the maps $\varrho_{n}: C \rightarrow F_{n}$ given in section 2, shows that the involutions $\tau_{i}$ take the following form in the model of $C$ given by $y^{2}=q(t)$ and $z^{2}=p(t)$ :

$$
\begin{aligned}
& \tau_{0}(t, y, z)=\left(\frac{6 t-5}{6(t-1)}, \frac{y}{6(t-1)^{2}}, \frac{z}{6(t-1)^{2}}\right), \\
& \tau_{1}(t, y, z)=\left(\frac{5(t-1)}{6 t-5}, \frac{5 y}{(6 t-5)^{2}}, \frac{5 z}{(6 t-5)^{2}}\right), \\
& \tau_{3}(t, y, z)=\left(\frac{5}{6 t}, \frac{5 y}{6 t^{2}}, \frac{5 z}{6 t^{2}}\right),
\end{aligned}
$$

$\tau_{2}(t, y, z)=(t, y,-z)$ and $\tau_{4}(t, y, z)=(t,-y, z)$. These can be used also to show the last Corollary.

Observe that the known points in $C(\mathbb{Q})$, corresponding to the points $[1: \pm 1: \pm 1: \pm 1: \pm 1]$ and $[1: \pm 3: \pm 5: \pm 7: \pm 9]$, give rise to the points in $C^{\prime(1,1)}(\mathbb{Q}(\sqrt{6}))$ with $t=1$ and in $C^{\prime(-1,1)}(\mathbb{Q}(\sqrt{6}))$ with $t=1 / 2$, respectively.

Now, in order to compute the points $\left(t, y_{1}, y_{2}, z_{1}, z_{2}\right)$ in $C^{\prime( \pm 1,1)}(\mathbb{Q}(\sqrt{6}))$ such that $t \in \mathbb{Q}$, we consider the following natural genus one quotients of $C^{\prime( \pm 1,1)}$ defined by

$$
H_{i, j}^{( \pm)}: \pm w^{2}=q_{i}(t) p_{j}(t)
$$

for $i, j=1,2$. We have four of them for any sign, corresponding in fact to the factors of a natural genus 4 quotient of any of the curves $C^{\prime( \pm 1,1)}$, which is defined over $\mathbb{Q}$.

Hence, we only need to compute, the points

$$
\left\{(t, w) \in H_{(i, j)}^{( \pm 1,1)} \quad: t \in \mathbb{Q} \text { or } t=\infty\right\}
$$

for some $(i, j)$, and we are done. But this can be done by using the elliptic Chabauty method.

The following diagram illustrates all the curves and morphisms involved in our problem:

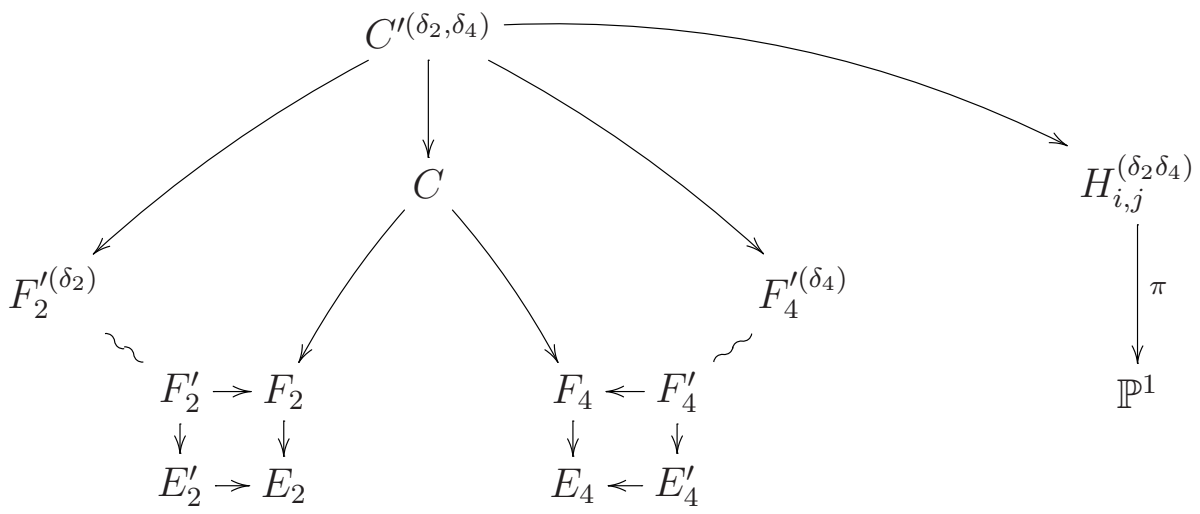

\section{An Elliptic Chabauty Argument}

Our aim in this section is to compute, for any choice of a sign \pm , all the $\mathbb{Q}(\sqrt{6})$-rational points in some of the curves $H_{i, j}^{ \pm}: \pm w^{2}=q_{i}(t) p_{j}(t)$ such that $t \in \mathbb{Q}$. We will be able to do these once we have that the jacobians 
of the curves have rank 0 or 1 over $\mathbb{Q}(\sqrt{6})$, a condition coming from the Chabauty technique.

Since we only need to show this result for just one $(i, j)$, we will do it for $(1,1)$ for both signs. This choice is not totally arbitrary, since one can show that all the cases with $j=2$ do have rank 2 , hence they do not fulfill the necessary conditions.

We will denote by $H^{ \pm}=H_{1,1}^{ \pm}$, which are the genus one curves defined over $\mathbb{Q}(\sqrt{6})$ by the equations

$$
\pm w^{2}=q_{1}(t) p_{1}(t)=(5+2 \sqrt{6})\left(6 t^{2}+2 \sqrt{6} t+5-6 \sqrt{6}\right)\left(6 t^{2}-4 t-1\right) .
$$

Lemma 7. Consider the points $P_{ \pm}^{+}:=(1, \pm 1) \in H^{+}(\mathbb{Q}(\sqrt{6}))$ and $P_{ \pm}^{-}:=$ $(1 / 2, \pm(-2 \sqrt{6}-3) / 2) \in H^{-}(\mathbb{Q}(\sqrt{6}))$. Then, the curves $H^{ \pm}$are isomorphic over $\mathbb{Q}(\sqrt{6})$ to their corresponding jacobians $J^{ \pm}:=\operatorname{Jac}\left(H^{ \pm}\right)$, which are given by the equations

$$
\begin{aligned}
J^{+}: y^{2}+(-2 \sqrt{6}-10) x y+ & (38 \sqrt{6}+22) y= \\
& x^{3}+(-24 \sqrt{6}-34) x^{2}+(448 \sqrt{6}+1253) x
\end{aligned}
$$

and

$$
\begin{aligned}
J^{-}: y^{2}+(42 \sqrt{6}+422) x y+(-291822 \sqrt{6}-113902) y= \\
x^{3}+(-8076 \sqrt{6}-33466) x^{2}+(67635708 \sqrt{6}+141575953) x,
\end{aligned}
$$

by isomorphisms $\mu^{ \pm}: H^{ \pm} \rightarrow J^{ \pm}$sending the points $P_{+}^{ \pm}$to the zero point in $J^{ \pm}$. Moreover, $\mu^{ \pm}\left(P_{-}^{ \pm}\right)=-(0,0)$.

Finally, we have that a point $(t, w) \in H^{ \pm}(\mathbb{Q}(\sqrt{6}))$ verifies that $t \in \mathbb{Q}$ if and only if $\pi^{ \pm}(x, y) \in \mathbb{P}^{1}(\mathbb{Q})$, where $(x, y)=\mu^{ \pm}(t, w) \in J^{ \pm}(\mathbb{Q}(\sqrt{6}))$ denotes the corresponding image of $(t, w)$ and

$$
\pi^{+}(x, y)=\frac{y}{2 x+y} \quad \text { and } \quad \pi^{-}(x, y)=\frac{y}{300 x+2 y} .
$$

Proof. The first part is an standard computation (see for example [7, $\S 7.2 .3]$ ). The inverse of the maps $\mu^{ \pm}$are given by the maps $\nu^{ \pm}$defined as

$$
\nu^{+}(x, y):=\left(\frac{y}{2 x+y}, \frac{2 x^{3}+(-24 \sqrt{6}-34) x^{2}+(2 \sqrt{6}+10) x y-y^{2}}{2 x+y}\right)
$$

and

$$
\nu^{-}(x, y):=\left(\frac{y}{300 x+150 y}, \frac{-2(2 \sqrt{6}+3) x^{3}+(91160 \sqrt{6}+197310) x^{2}+(970 \sqrt{6}+1770) x y+(2 \sqrt{6}+3) y^{2}}{150^{3}(150 x+y)}\right) .
$$

The maps $\pi^{ \pm}$are the composition of the map $\nu^{ \pm}$and the map $H^{ \pm} \rightarrow \mathbb{P}^{1}$ sending $(t, w)$ to $t$.

Remark 8. Note that $j\left(J^{ \pm}\right)=140608 / 245 \in \mathbb{Q}$. Moreover, $J^{ \pm}$is isomorphic to the $\pm(\sqrt{6}-3)$-twist of the elliptic curve defined over $\mathbb{Q}$ given by the Weierstrass model $y^{2}=x^{3}+312 x-3008$ (which is 80640CU2 in Cremona's tables). 
Lemma 9. The elliptic curves $J^{ \pm}$have both rank 1 over $\mathbb{Q}(\sqrt{6})$. We have

$$
J^{ \pm}(\mathbb{Q}(\sqrt{6})) \supset S^{ \pm}=\left\langle T^{ \pm},(0,0)\right\rangle
$$

where

$T^{+}:=(2 \sqrt{6}-7,-16 \sqrt{6}-34)$ and $T^{-}:=(-462 \sqrt{6}-2767,301500 \sqrt{6}+699000)$ are 2 -torsion points and $(0,0)$ is of infinite order. The subgroups $S^{ \pm}$are of finite index not divisible by any prime $<14$.

Proof. This is shown by an standard 2-descent argument, either using Magma, Sage or PARI (see for example [7, §8.3]). The non divisibility condition of the index can be shown easily by proving that the point of infinite order $(0,0)$ in both cases is not a $p$-multiple of another point in $J^{ \pm}(\mathbb{Q}(\sqrt{6}))$ for any prime $p<14$.

Now, we are under the conditions to apply the Chabauty technique. We need to choose a prime $p$ of good reduction for $J^{ \pm}$, and also inert in $\mathbb{Q}(\sqrt{6})$ (the technique can also be used for split primes, but with a slightly distinct form, see for example [4]). Denote by $J_{p}^{ \pm}$the reduction modulo $p$ of $J^{ \pm}$, which is an elliptic curve over $\mathbb{F}_{p^{2}}:=\mathbb{F}_{p}(\sqrt{6})$, and by $\operatorname{red}_{p}: J^{ \pm}(\mathbb{Q}(\sqrt{6})) \rightarrow$ $J_{p}^{ \pm}\left(\mathbb{F}_{p^{2}}\right)$ the reduction map. Then the Elliptic Chabauty method will allow us to bound, for each point $R$ in $J_{p}^{ \pm}\left(\mathbb{F}_{p^{2}}\right)$, the number of points $Q$ in $J^{ \pm}(\mathbb{Q}(\sqrt{6}))$ such that $\operatorname{red}_{p}(Q)=R$ and such that $\pi^{ \pm}(Q) \in \mathbb{P}^{1}(\mathbb{Q})$. Denote this set of points by

$$
\Omega_{ \pm, p}(R):=\left\{Q \in J^{ \pm}(\mathbb{Q}(\sqrt{6})) \mid \pi^{ \pm}(Q) \in \mathbb{Q} \text { and } \operatorname{red}_{p}(Q)=R\right\} .
$$

Clearly, we have

$$
\left\{Q \in J^{ \pm}(\mathbb{Q}(\sqrt{6})) \mid \pi^{ \pm}(Q) \in \mathbb{Q}\right\}=\bigsqcup_{R \in J_{p}^{ \pm}\left(\mathbb{F}_{p^{2}}\right)} \Omega_{ \pm, p}(R),
$$

for any choice of an inert good reduction prime $p$. So, if we compute these sets $\Omega_{ \pm, p}(R)$ for some choice of the prime $p$ and for all $R$, we have computed the sets we are interested on.

We will choose the primes $p=11$ and $p=13$, depending of the sign of the case considered.

Proposition 10. Denote by $p_{+}=11$ and $p_{-}=13$. Then $\Omega_{ \pm, p_{ \pm}}(\widetilde{R}) \neq \emptyset$ if and only if $\widetilde{R}=O$ or $\widetilde{R}=-(0,0)$.

Proof. First of all, observe that $\Omega_{ \pm, p_{ \pm}}(O) \neq \emptyset$ and $\Omega_{ \pm, p_{ \pm}}(-(0,0)) \neq \emptyset$ since they contain the points $O$ and $-(0,0)$, respectively.

In order to show the remaining subsets are empty, we will use arguments modulo $p_{ \pm}^{m}$ for various powers of $p_{ \pm}$. Denote by $\mathcal{O}$ the ring of integers of $\mathbb{Q}(\sqrt{6})$, by $\mathcal{J}$ the Néron model of $J$ over $\mathcal{O}$ and by $\pi_{p_{ \pm}^{n}}^{ \pm}: \mathcal{J}_{\mathcal{O} / p_{ \pm}^{n} \mathcal{O}}^{ \pm} \rightarrow \mathbb{P}^{1}$ the reduction modulo $p_{ \pm}^{n}$ of the map $\pi^{ \pm}$, which is a well-defined map of schemes 
over $\mathcal{O} / p_{ \pm}^{n} \mathcal{O}$. Observe that for good reduction primes $p$ as we have, the scheme $\mathcal{J}_{\mathcal{O} / p^{n} \mathcal{O}}^{ \pm}$is an abelian scheme.

First we work modulo $p_{ \pm}$. We get that $(0,0)$ has order 8 in $J_{11}^{+}\left(\mathbb{F}_{11^{2}}\right)$ and has order 12 in $J_{13}^{-}\left(\mathbb{F}_{13^{2}}\right)$. Since the point $(0,0)$ is not divisible by 2 and 3 in $J^{ \pm}(\mathbb{Q}(\sqrt{6}))$ as shown in lemma 9, we get in both cases that $\operatorname{red}_{p_{ \pm}}\left(S^{ \pm}\right)=\operatorname{red}_{p_{ \pm}}\left(J^{ \pm}(\mathbb{Q}(\sqrt{6}))\right)$, so we can work with the subgroup $S^{ \pm}$.

One easily computes that the only points $R$ in $\operatorname{red}_{p_{ \pm}}\left(S^{ \pm}\right)$such that $\pi_{p_{ \pm}}^{ \pm}(R) \in \mathcal{O} / p_{ \pm} \mathcal{O}=\mathbb{F}_{p_{ \pm}^{2}}$ are, in each case, the points

$$
O,-(0,0), T^{+}+2(0,0), T^{+}-3(0,0) \in \operatorname{red}_{p_{+}}\left(S^{+}\right)
$$

and

$$
O,-(0,0), 4(0,0),-5(0,0) \in \operatorname{red}_{p_{-}}\left(S^{-}\right) .
$$

Since $\Omega_{ \pm, p_{ \pm}}(R)$ is obviously empty if $\pi_{p_{ \pm}}^{ \pm}(R)$ is not in $\mathbb{F}_{p_{ \pm}^{2}}$, we only need to show that $\Omega_{+, p_{+}}(R)=\emptyset$ if $R=T^{+}+2(0,0)$ or $T^{+}-3(0,0)$, and that $\Omega_{-, p_{-}}(R)=\emptyset$ if $R=4(0,0)$ or $-5(0,0)$.

We start with the + case. In this case one computes all the points in $\mathcal{J}_{\mathcal{O} / 11^{2} \mathcal{O}}^{+}$which are equal to $R=T^{+}+2(0,0)$ or to $T^{+}-3(0,0)$ modulo 11 (there are 22 of them), and then we compute their images by $\pi_{11^{2}}^{+}$. But for any of these 22 points, the image is not in $\mathbb{Z} / 11^{2} \mathbb{Z} \hookrightarrow \mathcal{O} / 11^{2} \mathcal{O}$, hence $\Omega_{+, p_{+}}(R)=\emptyset$ for both points.

Finally, the - case is done similarly, but one needs to work modulo $13^{3}$, since all the lifts to $13^{2}$ do have image in $\mathbb{Z} / 13^{2} \mathbb{Z}$ with respect to the map $\pi_{13^{2}}^{+}$. When working modulo $13^{3}$, the total number of points to be considered is $2 \cdot 13^{2}$.

Proposition 11. The sets $\Omega_{ \pm, p_{ \pm}}(O)$ and $\Omega_{ \pm, p_{ \pm}}(-(0,0))$ contain, for any sign, only one point.

Proof. We use the Chabauty argument. First of all, recall that the order of $(0,0)$ modulo $p_{ \pm}$is either $m_{+}:=8$ in the + case and $m_{-}:=12$ in the - case. Hence, any point in $\Omega_{ \pm, p_{ \pm}}(R)$ must be of the form $R+n\left(m_{ \pm}(0,0)\right)$ for some $n \in \mathbb{Z}$. Since in both cases, $R=O$ and $R=-(0,0)$, we do have that $R$ is in $\Omega_{ \pm, p_{ \pm}}(R)$, we want to show that the only solution is $n=0$ in all cases. We will work with an argument modulo $p_{ \pm}^{2}$.

We will use the expression of the $z$-coordinate of the point $(x, y)$ (with respect to the given equation of $J^{ \pm}$) to mean $z:=-x / y$ (as a point in $\mathbb{P}^{1}$ ).

Let us denote by $z_{ \pm, p}$ the $z$-coordinate of the point $m_{ \pm}(0,0)$ modulo $p_{ \pm}^{2}$. We get $z_{+, 11}=11-55 \sqrt{6} \in \mathcal{O} / 11^{2} \mathcal{O}$ and $z_{-, 13}=26-39 \sqrt{6} \in \mathcal{O} / 13^{2} \mathcal{O}$. Because we are working modulo $p_{ \pm}^{2}$, we have

$$
z-\operatorname{coord}\left(n\left(m_{ \pm}(0,0)\right)\right)=n z_{ \pm, p_{ \pm}} \quad\left(\bmod p_{ \pm}^{2} \mathcal{O}\right)
$$

(a fact that can be proved using the formal logarithm and exponential of the elliptic curves $J^{ \pm}$). 
Now, we can express the function $\pi_{ \pm}(P)$ at any point $P$ as a power series in the $z$-coordinate of $\mathrm{P}$. We get, for the + case, that

$$
\pi_{+}(z)=1+2 z+4 z^{2}+8 z^{3}+O\left(z^{4}\right)
$$

and for the - case, that

$$
\pi_{-}(z)=1 / 2+75 z+11250 z^{2}+1687500 z^{3}+O\left(z^{4}\right) .
$$

First we treat the point $O$. We have that $\pi_{ \pm}\left(n\left(m_{ \pm}(0,0)\right)\right)$ can be expressed as a power series $\Theta(n)$ in $n$ with coefficients in $\mathbb{Q}(\sqrt{6})$. We express this power series as $\Theta(n)=\Theta_{0}(n)+\sqrt{6} \Theta_{1}(n)$, with $\Theta_{i}(n)$ now being a power series with coefficients in $\mathbb{Q}$. Then $\pi_{ \pm}\left(n\left(m_{ \pm}(0,0)\right)\right) \in \mathbb{Q}$ for some $n \in \mathbb{Z}$ if and only if $\Theta_{1}(n)=0$ for that $n$. Observe also that, since $\pi_{ \pm}(O) \in \mathbb{Q}$, we will get that $\Theta_{1}(0)=0$, so $\Theta_{1}(n)=j_{1} n+j_{2} n^{2}+j_{3} n^{3}+\cdots$. To conclude, we will use Strassmann Theorem: if the $p_{ \pm}$-adic valuation of $j_{1}$ is strictly smaller that the $p_{ \pm}$-adic valuation of $j_{i}$ for any $i>1$, then this power series has only one zero at the $p$-adic ring $\mathbb{Z}_{p_{ \pm}}$, and this zero is $n=0$. In fact, one can easily shown that this power series verifies that the $p_{ \pm}$-adic valuation of $j_{i}$ is always greater or equal to $i$, so, if we show that $j_{1} \not \equiv 0\left(\bmod p_{ \pm}^{2}\right)$ we are done.

Since the $z$-coordinate of $m_{ \pm}(0,0)$ is congruent to 0 modulo $p_{ \pm}$, to compute $\pi_{ \pm}\left(z\right.$-coord $\left.\left(n\left(m_{ \pm}(0,0)\right)\right)\right)$ modulo $p_{ \pm}^{2}$ we only need the power series up to degree 1 . We get

$$
\pi_{+}\left(z-\operatorname{coord}\left(n\left(m_{+}(0,0)\right)\right)\right)=(22-44 \sqrt{6}) n+1 \quad\left(\bmod 11^{2}\right)
$$

and

$$
\pi_{-}\left(z-\operatorname{coord}\left(n\left(m_{-}(0,0)\right)\right)\right)=(-78-52 \sqrt{6}) n-84 \quad\left(\bmod 13^{2}\right) .
$$

Hence, for the + case, $\Theta_{1}(n)=-44 n$ modulo $11^{2}$, thus the valuation of $j_{1}$ is 1 , and we are done. Similarly, for the - case, $\Theta_{1}(n)=-52 n$ modulo $13^{2}$, and again we are done.

In order to consider the other point $-(0,0)$ one can either compute directly $\pi_{ \pm}\left(z\right.$-coord $\left.\left(-(0,0)+n\left(m_{ \pm}(0,0)\right)\right)\right)$ as a power series in $n$ and apply the same type of arguments, or observe that there is an involution in $J^{ \pm}$ (as a genus 1 curve) that interchanges the points $O$ and $-(0,0)$ and preserves the function $\pi_{ \pm}$; it corresponds to the hyperelliptic involution on $H^{ \pm}$ sending $(t, w)$ to $(t,-w)$.

Hence, by using the results just proved, we obtained finally the following.

Corollary 12. The only points $\left(t_{ \pm}, w\right) \in H^{ \pm}(\mathbb{Q}(\sqrt{6}))$ with $t_{ \pm} \in \mathbb{Q}$ are the points with $t_{+}=1$ and with $t_{-}=1 / 2$.

\section{Proof of the theorem 1}

By using the results proved in the last two sections, we have finally computed all the rational points in the curve $C$. 
Theorem 13. $C(\mathbb{Q})=\{[ \pm 1: \pm 1: \pm 1: \pm 1: \pm 1],[ \pm 1: \pm 3: \pm 5: \pm 7:$ $\pm 9]\}$.

Proof. We review here briefly the steps we followed. By using Corollary 5, we have that, for any point $P \in C(\mathbb{Q})$, there exists a sign \pm and an involution $\tau \in \Upsilon$ of $C$ such that

$$
\tau(P) \in \chi^{(( \pm 1,1))}\left(\left\{\left(t, y_{1}, y_{2}, z_{1}, z_{2}\right) \in C^{\prime(( \pm 1,1))}(\mathbb{Q}(\sqrt{6})): t \in \mathbb{Q}\right\}\right) .
$$

Moreover, the points $[ \pm 1: \pm 1: \pm 1: \pm 1: \pm 1]$ and $[ \pm 1: \pm 3: \pm 5: \pm 7: \pm 9]$ come, respectively, from the points in $C^{\prime((1,1))}$ with $t=1$ and the points in $C^{\prime((-1,1))}$ with $t=1 / 2$.

After that, we consider the genus one quotients $H^{ \pm}$of the curves $C^{\prime(( \pm 1,1))}$ with quotient maps defined by $\left(t, y_{1}, y_{2}, z_{1}, z_{2}\right) \mapsto\left(t, y_{1} z_{1}\right)$. We get that the points $\left(t, y_{1}, y_{2}, z_{1}, z_{2}\right) \in C^{\prime(( \pm 1,1))}(\mathbb{Q}(\sqrt{6}))$ go to points $(t, w) \in H^{ \pm}(\mathbb{Q}(\sqrt{6}))$. Finally, by Corollary [12, we have that the only points in $H^{+}$with $t \in \mathbb{Q}$ are the ones with $t=1$, and the only points in $H^{-}$with $t \in \mathbb{Q}$ are the ones with $t=1 / 2$. This proves the result.

Proof of Theorem 1. If $N$ is odd, then translating by $-r-(N-1) / 2$ we can suppose $r=(N-1) / 2$. Thus, $b=0$ and $f(x)=a x^{2}+c$ is symmetric with respect the axis $x=0$. In the even case, we can apply the translation by $-r-N / 2$, and we can suppose that $r=-N / 2$. Thus, $b=a$ and $f(x)=a\left(x^{2}+x\right)+c$ is symmetric with respect the axis $x=-1 / 2$. Now, the existence of $f(x)$ is equivalent to the existence of $x_{k} \in \mathbb{Z}$ such that $f(k)=x_{k}^{2}, k=0,1, \ldots, s_{N}$, where $s_{N}=(N-1) / 2$ or $s_{N}=N / 2-1$ depending on $N$ is odd or even, respectively. Note that for $N \leq 4$ is trivial to prove that there are infinitely many non-square quadratic satisfying the hypothesis. If $N=5$ (resp. $N=6$ ), then they satisfy $3 x_{0}^{2}+x_{2}^{2}=4 x_{1}^{2}$ (resp. $\left.2 x_{0}^{2}+x_{2}^{2}=3 x_{1}^{2}\right)$, that it is a conic in $\mathbb{P}^{2}$ with infinitely rational points. If $N=7$ (resp. $N=8$ ), then they satisfy $3 x_{0}^{2}+x_{2}^{2}=4 x_{1}^{2}$ and $8 x_{0}^{2}+x_{3}^{2}=9 x_{1}^{2}$ (resp. $2 x_{0}^{2}+x_{2}^{2}=3 x_{1}^{2}$ and $5 x_{0}^{2}+x_{3}^{2}=6 x_{1}^{2}$ ), which is isomorphic to the elliptic curve $y^{2}=x(x-5)(x+27)$ (resp. $\left.y^{2}=x(x-12)(x-15)\right)$ and it is denoted by 30A2 (resp. 360E2) at Cremona's table with Mordell-Weil group isomorphic to $\mathbb{Z} / 2 \mathbb{Z} \oplus \mathbb{Z} / 6 \mathbb{Z}$ (resp. $\mathbb{Z} / 2 \mathbb{Z} \oplus \mathbb{Z} / 2 \mathbb{Z} \oplus \mathbb{Z}$ ). Therefore if $N \geq 7$ odd the proof of the theorem is finished. If $N=8$, since the rank of the underlying elliptic curve is non zero then there are infinitely many non-square quadratic polynomials satisfying the hypothesis. The remaining case to finish the proof is when $N \geq 10$ even. The characterization given at section 2, give us that if $N=10$ then any quadratic polynomial $f(x) \in \mathbb{Z}$ satisfying the hypothesis of the theorem correspond to a point $P \in C(\mathbb{Q})$. At Theorem 13 we have proved that the unique points at $C(\mathbb{Q})$ are $[ \pm 1: \pm 1: \pm 1: \pm 1: \pm 1]$, $[ \pm 1: \pm 3: \pm 5: \pm 7: \pm 9]\}$ that correspond to the constant polynomials and to $f(x)=(2 x+1)^{2}$ respectively.

Data: All the Magma sources are available from the first author webpage. 


\section{REFERENCES}

[1] D. Allison, On certain simultaneous Diophantine equations, Math. Colloq. Univ. Cape Town 11 (1977), 117-133.

[2] D. Allison, On square values of quadratics, Math. Proc. Cambridge Philos. Soc. 99 (1986), 381-383.

[3] A. Bremner, On square values of quadratics. Acta Arith. 108 (2003), no. 2, 95-111.

[4] N. Bruin, Chabauty methods using elliptic curves. J. Reine Angew. Math. 562 (2003), $27-49$.

[5] N. Bruin, E. V. Flynn, Towers of 2-covers of hyperelliptic curves, Trans. Amer. Math. Soc. 357 (2005), 4329-4347.

[6] J.J. Cannon, W. Bosma (EDs.), Handbook of Magma Functions. Edition 2.15-6 (2009).

[7] H. Cohen, Number Theory, Springer-Verlag, GTM 239-240.

[8] K.R. Coombes, D. Grant, On heterogeneos spaces, Journal of the London Mathematical Society, series 2, 40 (3) (1989),385-397

[9] J. E. CREmona, Elliptic curve data. Available on http://www.warwick.ac.uk/ masgaj/ftp/data/, 2008.

[10] E.V. Flynn, J.L. Wetherell, Covering collections and a Challenge Problem of Serre, Acta Arith. 98 (2001), 197-205

[11] E. GonzÁlez-JimÉnez, X. XARles, Five squares in arithmetic progression over quadratic fields. arXiv: 0909.1663.

[12] J-H. Silverman, The Arithmetic of Elliptic Curves, Springer-Verlag, GTM 106, 1986 .

[13] W. Stein et AL., Sage: Open Source Mathematical Software (Version 4.3), The Sage Group, 2009, http://www.sagemath.org.

[14] X. XARLEs, Squares in arithmetic progression over number fields. arXiv: 0909.1642 [15] J.L. Wetherell, Bounding the Number of Rational Points on Certain Curves of High Rank, PhD Dissertation (1997), University of California at Berkeley.

Universidad Autónoma de Madrid, Departamento de Matemáticas and Instituto de Ciencias Matemáticas (CSIC-UAM-UC3M-UCM), MAdRid, Spain E-mail address: enrique.gonzalez.jimenez@uam.es

Departament de Matemàtiques, Universitat Autònoma de Barcelona, 08193 Bellaterra, Barcelona, Spain

E-mail address: xarles@mat.uab.cat 